\title{
Mediastinal Sarcoma
}

National Cancer Institute

\section{Source}

National Cancer Institute. Mediastinal Sarcoma. NCI Thesaurus. Code C6606.

A rare sarcoma that arises from the mediastinum. Examples include liposarcoma, leiomyosarcoma, and angiosarcoma. 